\title{
Construction of low cost remote controlled slit lamp (R/C S/L) in order to provide teleophthalmology services to rural areas and islands
}

\section{Opinion}

We are all aware of the impact the financial crisis had on health care systems and on the quality and cost of life of the patients in general. Especially for Greece, a distinct geographically country of the European Union with 227 inhabited islands, where primary health care centers do not provide Ophthalmological services. Commuting of patients to specialized Eye Care centers or Hospitals is expensive, time consuming and not possible all the days of the week. Boat connections with the mainland are scarce, once every other day in some places, and cancelled in the case of rough sea. Since the beginning of the crisis, the cost of fuel and taxation has been passed to the community, and for people living on a pension of $€ 300$, traveling is difficult, particularly if they have chronic health problems. Cost is increased by the fact that most of them will be accompanied by younger relatives, leading also to lost human hours of productive age people in commuting. Teleophthalmology can provide services to remotely located people, providing emergency, screening and follow-up ophthalmological services. As much of the portable technology is already commercially available, from refraction, to clinical examination, and fundus photography, we had to complete with the gold standard of any ophthalmological examination, the slit lamp.

In response to that need, we constructed a remote controlled slit lamp (R/C S/L) Figure 1 which permits the telexamination of patients located in remote Islands. As the project was financed personally, we had to keep the cost as low as possible. A used Zeiss ${ }^{\circledR}$ Slit lamp model $30 \mathrm{M}$ was purchased from eBay. The necessary motors and controllers in order motorize three axis, back and forth, left and right of the Slit lamp, and rotation of the lighting column for slit angulation were purchased from Advanced Microsystems ${ }^{\circledR}$. In the initial phase of construction only those three axis were motorized in order to get preliminary data to support further investment. For capturing and streaming of video, we used two different models of Apple ${ }^{\circledR}$, iPhone $4 \mathrm{~S}$ and $5 \mathrm{~S}$, with an advanced adapter with optics Figure 2. For streaming we used two different streaming protocols, Apple Facetime $\odot$ Figure 3-5 and Skype HD $\odot$. Both of them permit encrypted video streaming of the images from the slit lamp, but also the direct commination of the patient with the doctor during the examination through the iPhone speaker. The iPhone and the motor controllers connect with the internet through a $3 \mathrm{G} / 4 \mathrm{G}$ mobile connection, so no infrastructure is necessary in the primary health center. In the other side of the cloud, the examining doctor uses a computer to place a video call to the iPhone and a joystick to control the motors as video quality in both streaming protocols depends on bandwidth available at the moment. $4 \mathrm{G}$ connections are faster than $3 \mathrm{G}$ but do not cover all of Greece. Signal stability is an issue, as signal strength, and therefore video quality depends on many factors. Both protocols downgrade automatically video quality when the bandwidth is low, and the user does not have any options for bandwidth use, as
Volume I Issue 4 - 2014

\author{
lordanis Chatziangelidis \\ Ophtalmiatrion, Athens Eye Hospital, Greece
}

Correspondence: lordanis Chatziangelidis, Ophtalmiatrion, Athens Eye Hospital, Pirgou 43 45, Glifada 16675, Athens, Greece, Tel +306947727228, Email lides@me.com

Received: November II, 2014 | Published: December 10, 2014

for example prioritize video quality, over frames per second or audio quality.

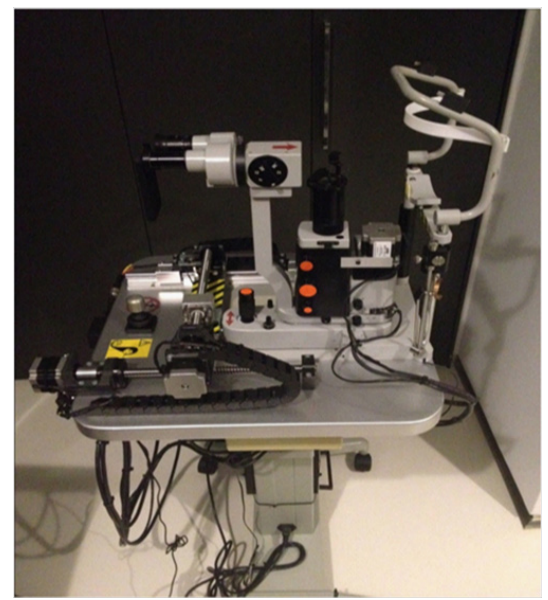

Figure I Remote Controlled Slit Lamp, R/C S/L with the iphone adapter and Volk Steady Mount attached.

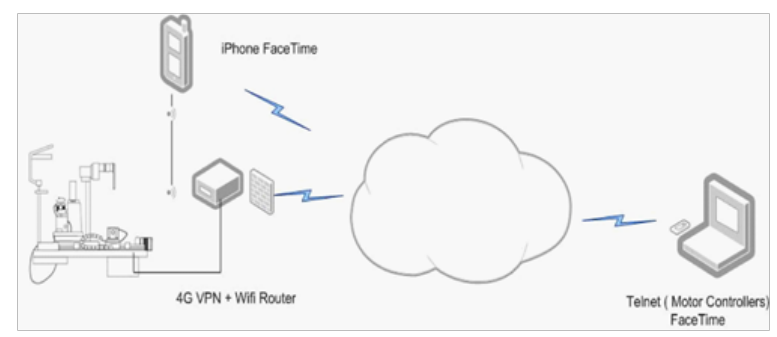

Figure 2 Network Setup.

Before the examination the makes an interview with the patient, taking notes on medical, family and ophthalmological history of the patient and his presenting complain. With the use of special free software for the iphoneas EyeHandBook $\odot$, it is possible to examine the patient for near and color vision and for Amsler Grid. 
Docking the iPhone to the slit lamp adapter and the slit lamp telexamination can begin. For testing purposes, we examined volunteer patients from the Emergency Department of our hospital, after they were examined by the doctor in service. Then the findings of the remote controlled slit lamp were compared with those of the doctor of the emergencies department. Preliminary data are encouraging. Hyphema, Hypopyon, Band Keratopathy, Corneal Oedema, Corneal Erosions (with use of fluroscein), Cornal Filaments, Corneal Opacification or Infiltrations, Ectasia and Tyndal $>2+$, Van Henrick angle classification, disorders of the Eyelids, Blepharitis and Cataract (after mydriasis) is possible to detect.

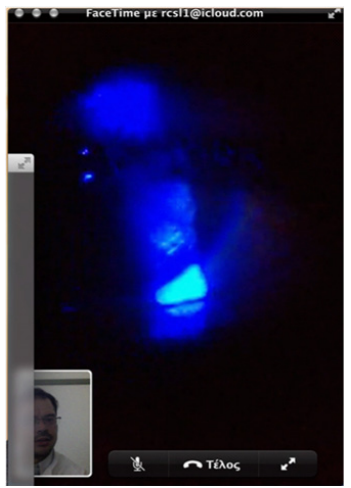

Figure 3 Apple ${ }^{\circ}$ Facetime Screenshot of Corneal Abrasion after instillation of Fluorescein.

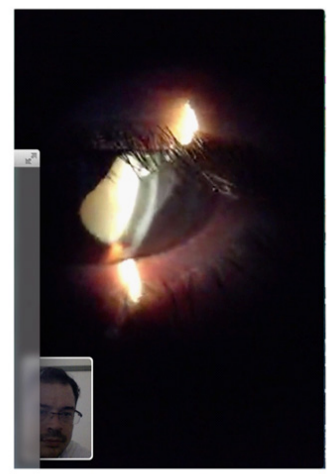

Figure 4 Apple ${ }^{\circledR}$ Facetime Screenshot of hyper mature cataract after instillation of midriatics.

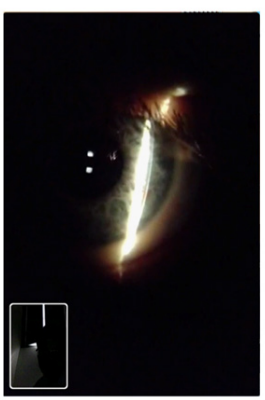

Figure 5 A Apple Facetime Screenshot of a shallow anterior chamber.
Fundus examination is possible with the use of the attached Volk ${ }^{\circledR}$ Steady Mount and a 75D lens, but it is time consuming as continuous adjustments should be made on the position of the slit lamp. A portable fundus camera like the Volk picture, or the WeltchAllyn Ophthalmoscope with the iphone adapter, can provide a picture directly to the examining ophthalmologist, for diagnosis of an acute illnessor staging a chronic illness as diabetic retinopathy. A nurse or a general doctor will be necessary at the remote health care center for the accommodation of the patient on the slit lamp, or for installation of midriatics or fluorescein. With the use of accessory devices as mobile tonometer's, like the iCare device, and portable auto refractors (Adaptica, Volk etc.) screening and diagnosis of refractive problems, strabismus and amblyopia can be performed remotely. As many of the smallest islands do not have a pharmacy, therapy will be provided by the primary health care center after a diagnosis is made by the ophthalmologist examining remotely. In the next stage of construction we plan to motorize the magnification dial, slit width, height and illumination intensity, giving the ophthalmologist full control over the slit lamp. We believe that with full control over the slit lamp, even Tyndal $1 / 2+$, will be possible to detect.

As this project is self financed, and no investors or industry support are available, we believe that the project will be completed within few months. Finally we believe that a remote controlled slit lamp is possible and can provide specialized health care services in remote primary health centers, decreasing the commuting burden for the islanders, provide services for the tourists, and generally improving the quality of life of people living or visiting those small and beautiful islands. Widening the picture, the service could be used in remote areas around Europe or in the world, in offshore petroleum drilling platforms, in commercial long range cruising ships, as adjuvant to the European Space Agency's Concordia base in Antarctica, or even at the International Space Station. Everywhere, where specialized Ophthalmological Services are needed and commuting is difficult or impossible.

\section{Acknowledgments}

None.

\section{Conflicts of interest}

Author declares that there is no conflict of interest. 